\title{
Research Progress on the Relationship between Intestinal Flora and Mental and Psychological Diseases
}

\author{
Huang Tao', Chun-Ru Wang2 ${ }^{*}$, Jun-Cheng Guo ${ }^{3}$, Min Guo ${ }^{1}$ \\ ${ }^{1}$ Hainan General Hospital, Haikou, China \\ ${ }^{2}$ People's Hospital of Qionghai City, Qionghai, China \\ ${ }^{3}$ Central South University Xiangya School of Medical Affiliated Haikou Hospital, Haikou, China \\ Email: *wchunru@163.com
}

How to cite this paper: Tao, H., Wang, C.-R., Guo, J.-C. and Guo, M. (2020) Research Progress on the Relationship between Intestinal Flora and Mental and Psychological Diseases. Advances in Microbiology, 10, 295-305.

https://doi.org/10.4236/aim.2020.106021

Received: June 4, 2020

Accepted: June 27, 2020

Published: June 30, 2020

Copyright $\odot 2020$ by author(s) and Scientific Research Publishing Inc. This work is licensed under the Creative Commons Attribution International License (CC BY 4.0).

http://creativecommons.org/licenses/by/4.0/

\begin{abstract}
The effects of microorganisms on the central nervous system are manifested in both health and disease states. The symbiotic microorganisms in the human gut have certain effects on the brain development before and after birth. Chronic inflammation in patients with irritable bowel syndrome (IBS), psychiatric disorders such as PTSD, anxiety disorders, etc., is likely to be associated with an imbalance in gut flora. Intestinal flora regulates the central nervous system mainly through nerve, endocrine, metabolic and immune pathways. The mechanisms involved in the influence of intestinal flora on the central nervous system are diverse, so it is necessary to explore the role of intestinal flora in mental and psychological diseases.
\end{abstract}

\section{Keywords}

Intestinal Microbes, Psychology, Disease Influence

\section{Introduction}

The direct link between the brain and gut flora may seem implausible, but the use of antibiotics and probiotics in the clinic has shown that gut flora can influence brain function. In decompensated cirrhosis, the use of antibiotics and probiotics can alleviate or reverse hepatic encephalopathy [1] [2]; in addition, in patients with gastrointestinal diseases associated with psychological disorders, such as irritable bowel syndrome, the imbalance of intestinal flora is common, and the use of probiotics can improve gastrointestinal symptoms and their accompanying depressive and anxiety symptoms [3]. At the same time, animal experi- 
ments have also proved that intestinal flora is involved in the occurrence and development of neurological diseases caused by immune disorders, and can regulate brain development in rats with multiple sclerosis model [4]. Therefore, this emerging field of research not only gives us a better understanding of this range of diseases, but also provides us with new ideas to find treatments for these diseases. Based on the existing animal experiments, this paper mainly discusses how the intestinal flora regulates the brain activity and leads to the occurrence and development of mental and psychological diseases. At the same time, the limited relevant clinical research is also reviewed, so as to provide new ideas for the research and treatment of mental and psychological diseases.

There is a complex ecosystem of microorganisms in the human gut, including bacteria, fungi, archaea and viruses. These microorganisms are collectively called intestinal microbiota [5]. The human intestinal microflora is mainly composed of Firmicutes and Bacteroidetes, which are dominated by clostridium. In addition, actinomycetes and proteobacteria also occupy a certain amount of intestinal microflora. The number, species and distribution of intestinal microbiota are related to the age, sex, diet, medication, infection and environment of the individual [6] [7]. The intestinal microbiota colonized in a patchy form, forming a biota zone, there is no obvious distribution pattern. Although the distribution of intestinal microflora varies greatly among host individuals, the higher-order taxa are similar, so the random distribution needs to be further explored. A large number of bacteria inhabit the human intestinal tract. The total number of bacteria colonized in the intestinal tract of an average person is estimated to be about 1000 kinds, and the total number of genes is about several hundred times that of human genes, which has an important impact on human life activities [8] [9]. Colonization of the host intestinal flora plays a very important role in the physiological functions of the host, including the uptake of energy from food, production of important metabolites, promotion of the development and maturation of the immune system, and protection of the host against pathogen infection [10]. Previous studies have focused on the relationship between intestinal bacteria and digestive diseases, such as irritable bowel syndrome and infectious diarrhea. There are also many reports that changes in intestinal flora are closely related to diabetes, arthritis, and even tumors [11] [12] [13] [14] [15]. Recent studies have shown that intestinal flora has a huge regulatory effect on the development and behavior of host brain [16] [17] [18]. Under pathological conditions, the balance of intestinal microenvironment is broken, resulting in the imbalance of bacterial flora. The team, led by Professor Zhao Liping, found changes in the number of certain bacteria in the intestines of diabetic model animals [19]. It suggests that some kinds of lactobacillus in the intestinal tract may be involved in the development of diabetes. The change of flora is not only a consequence of diabetes, but also a possible cause of diabetes. Intestinal microecological imbalance has been found to be closely related to irritable bowel syndrome, inflammatory bowel disease, asthma, obesity, diabetes, liver cirrhosis and 
other pathological conditions [20] [21] [22]. In recent years, studies have found that intestinal flora plays an important role in the human immune, neuroendocrine and other physiological functions and the occurrence of many diseases [23] [24]. Recent research has revealed that gut flora interacts with the brain through the gut. Evidence suggests that gut flora plays an important role in regulating mood and brain activity. Whether it is the downstream regulation of the brain or the upstream regulation of the intestinal flora, it mainly plays a role through immunity, HPA axis and neurotransmitter [25]. Exactly how the gut flora is related to the brain is still being explored and it is speculated that increased permeability of the intestinal wall caused by stress may play an important role. The colonized intestinal flora and its products enter the circulatory system through the intestinal wall, thus activating the immune system and HPA axis in rats. Taking probiotics prevents translocation. Researchers have found evidence of intestinal bacterial translocation in the serum of people with mental illness, such as depression, which may be linked to abnormally activated immune systems and the HPA axis [26]. The findings suggest that gut flora may be a potential target for the treatment of mental illness. In particular, animal studies in recent years have shown that depression can cause changes in gut flora [27]. Meanwhile, the composition of intestinal flora of rats exposed to long-term stress was significantly different from that of the control group. In addition, Bailey [28] et al. exposed rats to social stress resulting in structural changes in the intestinal flora of the rats, which were observed early in the exposure period. Although these animal models confirm the effects of depression on the intestinal microecosystem, whether this relationship exists in people of different RACES and regions needs further confirmation. Recently, the correlation between intestinal microbiota and some mental and psychological diseases has become a new research hotspot.

\section{Intestinal Bacteria and Post-Traumatic Stress Disorder}

Post traumatic stress disorder (PTSD) is a type of stress related disorder in which sudden, threatening or catastrophic life events lead to delayed appearance and long-term persistence of individual clinical symptoms that are severe and greatly impair mental health. Studies have shown that the average incidence of PTSD due to different types of trauma is about $12 \%$ and can be as high as $80 \%$ [29]; epidemiological studies show that more than half of people with PTSD have comorbidities such as depression, other anxiety disorders, and substance abuse. In recent decades, with the rapid development of society and the accelerated pace of life, the frequent occurrence of various traumatic events, such as natural disasters, wars, terrorist incidents, traffic accidents, public safety incidents and so on, people are under increasing psychological stress, and the incidence of PTSD is on the rise. PTSD has become a serious public health and social problem. PTSD has attracted more and more attention and is one of the current research hotspots. Research on the pathogenesis of PTSD has focused on 
its association with genetic susceptibility, neuroendocrinology, neuroimmunology, biochemistry, and psychosocial environment [30] [31] [32] [33]. As an indicator of the activity of the hypothalamic-pituitary-adrenal axis (HPA), cortisol secretion regulates and reflects the physiological response of the body to fear and stress, which has become a consensus among experts. Recent studies have found that the development and maintenance of PTSD is closely related to functional changes in the HPA axis [34] [35]. Typically, exposure to stress triggers an emotional response, which activates the hypothalamus through a connection to the limbic system of the brain, triggering activity in the HPA axis. Corticotropin releasing hormone $(\mathrm{CRH})$ followed by paraventral hypothalamus neurons ACTS on the anterior pituitary and releases Adrenocorticotropin (ACTH) hormone. ACTH is transported to the adrenal cortex through peripheral blood circulation, which promotes the production and release of cortisol. This whole process is called forward feedback function of the HPA axis, while the process in which elevated cortisol levels inhibit the pituitary, hypothalamus, and hippocampus through a negative feedback mechanism is called negative feedback function of the HPA axis. The HPA axis should play an important role in pressure response and homeostasis maintenance. Other studies have found that urine and plasma dopamine (DA) levels are noted to be positively correlated with PTSD [36] [37], DA is a neurotransmitter closely associated with memory, and PTSD is at least partly a mental disorder associated with emotional memory, so the association between the DA system and PTSD symptoms is understandable. The researchers found that the different stages of conditioned fear memory formation are regulated by the DA system. Although these studies, along with related genetic studies and phenotypic polymorphism studies, provide some compelling evidence for the complexity of the pathogenesis of PTSD. However, a full, comprehensive and systematic understanding of the etiology and pathogenesis of PTSD is not yet available, and new related factors, especially important common links, need to be actively explored in order to obtain key measures to intervene in the development of PTSD.

The brain-gut axis is the bidirectional regulating axis of the interaction between the brain and the gastrointestinal tract, including central nervous system (CNS), autonomic nervous system (ANS), enteric nervous system (ENS), HPA axis and other structures. The functions of each part are coordinated. The gastrointestinal tract is the only organ controlled jointly by CNS, ANS and ENS in the body, which has both sensory function and motor function. It is called "emotional reactor". Gastrointestinal discomfort is often accompanied by emotional response, which, in turn, activates the neural activity of CNS related parts, transmits regulatory information down the brain-gut axis to the gastrointestinal tract, changes its dynamic and secretory functions, activates intestinal mucosal immunity, and affects intestinal mucosal barrier function. Irritable bowel syndrome patients are often accompanied by physical discomfort, anxiety, depression, fatigue and other symptoms, and some animal models of irritable bowel syndrome are also based on the brain-gut axis theory [38] [39] [40]. Some pa- 
tients with inflammatory bowel disease are associated with anxiety and depression, and the severity of anxiety and depression parallels the activity of the disease [41]. Most functional gastrointestinal patients were associated with HPA axis abnormality and ANS dysfunction. In patients with gastroesophageal reflux, there is a strong correlation between anxiety, depression and gastrointestinal symptoms such as heartburn, and psychological or antidepressant treatment is effective in some patients [42]. In psychiatric patients, depression and generalized anxiety disorders are often accompanied by gastrointestinal discomfort. These studies suggest that disorders of the brain-gut axis may play a role in the development of mental illness.

\section{Intestinal Bacteria and Autism and Depression}

Autism spectrum disorder (ASD) is a neurobehavioral developmental disorder associated with impaired social and verbal communication. Autism is a classic autism spectrum disorder. Studies have shown a link between gut flora and autism. The imbalance of intestinal flora may lead to the overproliferation of neurotoxin-producing bacteria and induce autism symptoms. Many patients with GENERALIZED anxiety disorder often see the gastroenterology department for gastrointestinal symptoms for the first time [43]; especially in children with autism, gastrointestinal symptoms such as nausea, vomiting, constipation, diarrhea and other common [44]. It has been reported that oral vancomycin has a certain effect on aggressive children with autism in the short term [45]. Changes in intestinal flora and specific intestinal symbiotic strains play a role in autism spectrum disorders [46]. Clostridium difficile is presumed to cause autism. In fact, two studies have found that in addition to $C$. difficile, autistic people have imbalances in other intestinal flora [47] [48] [49] [50] [51]. Autism patients have an imbalance between firmicutes and bacteroidetes [48]. It was found that bacteroidetes dominated the autistic patients and firmicutes dominated the healthy controls [49]. In addition, intestinal flora composition changes, such as bifidobacteria, lactobacillus. However, studies have cast doubt on the link between gut flora and autism. This difference may be due to sampling and detection techniques, while differences in the metabolic components of urine and faeces in autistic and healthy people may also lead to differences in the bacterial flora. Depression is a common mood disorder caused by neuropsychological or immune imbalance [52]. Probiotics can relieve depressive symptoms in animal models of depression. Lactic acid bacteria are also thought to have antidepressant properties. Lactobacillus rhamnosus and Lactobacillus helveticus can relieve depressive symptoms caused by mother and child separation by restoring cortisol levels [53]. Similarly, Lactobacillus rhamnosus has been shown to regulate cortisol and GABA receptors through the vagal pathway, thereby reducing depressur-related behavior [54]. Bifidobacterium also has potential antidepressant effects. Infant bifidobacteria can reduce depressive symptoms in rats that were forced to swim or separated from their mothers. These mechanisms may be associated with lo- 
wering inflammatory cytokines, regulating serotonin and central nervous system transmitters [55]. In rat models of myocardial infarction accompanied by depression, the probiotics Lactobacillus and Bifidobacterium have been shown to act as antidepressants by reducing inflammatory factors and rebuilding the intestinal wall membrane barrier [55]. In addition, intestinal microbial metabolites such as butyrate have been found to have antidepressant effects in animal experiments. In addition, the mixture of unsaturated fatty acid N. 3, Lactobacillus rhamnosus and Lactobacillus helveticus can also reverse the depressive symptoms in rats after myocardial infarction [55].

\section{Gut Bacteria and Anxiety}

Anxiety disorders are common neurological, endocrine and immune-based mood disorders. Exposure to stress can lead to reactions such as nervousness and anxiety, accompanied by activation of the HPA axis. Many diseases associated with tension and anxiety often have intestinal dysfunction, indicating abnormalities in neurotransmitters and immunity in the signaling of the brain's gut axis. Compared with the normal rats, the sterile rats showed more activity and less anxiety [56] [57]. This behavioral change is associated with reduced neurotransmitter receptors in the central nervous system and increased serotonin metabolism. Therefore, it can be speculated that intestinal flora plays a role in neurotransmitter regulation [58] [59]. Opportunistic pathogens in the gut can cause anxious behavior. Campylobacter jejuni infection can lead to anxious behaviors, mainly by inducing FOS protein production in the central nervous system and autonomic nervous system [60] [61]. However, probiotics can relieve anxiety symptoms. In animal studies, both bifidobacteria and lactobacillus were found to correct symptoms in anxious rat models. Intestinal flora can also affect the function of the HPA axis. The hyperactivity of HPA axis in sterile rats was accompanied by an increase in peripheral neurotransmitters and a decrease in brain-derived neurotrophic factors [62]. In addition, external stress can affect the composition of gut flora [63]. It was found that mother-child isolation resulted in decreased bacteroides and increased clostridium in the intestinal flora of rhesus monkeys, accompanied by an increase in peripheral inflammatory factors. Lactobacillus helveticus and Lactobacillus rhamnosus were administered to rats to relieve the psychological stress caused by water restriction and to reshape the integrity of the intestinal mucosal wall [64]. Lactobacillus farciminis also prevents an increase in intestinal permeability caused by stress and reduces serum cortisol levels [65]. Bifidobacterium longum can correct anxious behavior and restore BDNF level in the central nervous system of rats with infectious colitis through vagal nerve pathway [66]. Lactobacillus helveticus and Bifidobacterium longum have anti-anxiety effects in both animals and healthy people.

\section{Conclusion and Prospect}

More and more animal and human studies show the brain, the bowel, and the 
presence of microbial axes. Microbes regulate the central nervous system, immunity, and behavior in both disease and health. Although the interaction between gut microbes and the brain has been gradually recognized in recent years, the role of microbes in psychiatric diseases needs further study.

\section{Foundation Project}

Key Science and Technology Project of Hainan Province in 2018 (Approval Number: ZDYF2018140).

\section{Conflicts of Interest}

The authors declare no conflicts of interest regarding the publication of this paper.

\section{References}

[1] Kimer, N., Krag, A., Moiler, S., Bendtsen, F. and Gluud, L.L. (2014) Systematic Review with Meta-Analysis: The Effects of Rifaximin in Hepatic Encephalopathy. Alimentary Pharmacology \& Therapeutics, 40, 23-32.

https://doi.org/10.1111/apt.12803

[2] McGee, R.G., Bakens, A., Wiley, K., Riordan, S.M. and Webster, A.C. (2011) Probiotics for Patients Wim Hepatic Encephalopathy. The Cochrane Database of Systematic Reviews, 11, CD008716. https://doi.org/10.1002/14651858.CD008716.pub2

[3] Ford, A.C., Quigley, E.M., Lacy, B.E., et al. (2014) Efficacy of Prebiotics, Probiotics, and Synbiotics in Irritable Bowel Syndrome and Chronic Idiopathic Constipation: Systematic Review and Meta-Analysis. The American Journal of Gastroenterology, 109, 1547-1561. https://doi.org/10.1038/ajg.2014.202

[4] Bhargava, P. and Mowry, E.M. (2014) Gut Microbiome and Multiple Sclerosis. Current Neurology and Neuroscience Reports, 14, Article No.: 492. https://doi.org/10.1007/s11910-014-0492-2

[5] Eckburg, P.B., Bik, E.M., Bernstein, C.N., et al. (2005) Diversity of the Human Intestinal Microbial Floral. Science, 308, 1635-1638.

https://doi.org/10.1126/science.1110591

[6] Lozupone, C.A., Stombaugh, J.I., Gordon, J.I., Jansson, J.K. and Knight, R. (2012) Diversity, Stability and Resilience of the Human Gut Microbiota. Nature, 489, 220-230. https://doi.org/10.1038/nature11550

[7] Hakansson, A. and Molin, G. (2011) Gut Microbiota and Inflammation. Nutrients, 3, 637-682. https://doi.org/10.3390/nu3060637

[8] Bravo, J.A., Julio-Pieper, M., Forsythe, P., et al. (2012) Communication between Gastrointestinal Bacteria and the Nervous System. Pharmacology, 12, 667-672. https://doi.org/10.1016/j.coph.2012.09.010

[9] Bonaz, B.L. and Bernstein, C.N. (2013) Brain-Gut Interactions in Inflammatory Bowel Disease. Gastroenterology, 144, 36-49. https://doi.org/10.1053/j.gastro.2012.10.003

[10] Round, J. and Mazmanian, S.K. (2009) The Gut Microbiota Shapes Intestinal Immune Responses during Health and Disease. Nature Reviews Immunology, 9, 313-323. https://doi.org/10.1038/nri2515

[11] Collins, S.M. (2014) A Role for the Gut Microbiota in IBS. Nature Reviews Gastro- 
enterology \& Hepatology, 11, 497-505. https://doi.org/10.1038/nrgastro.2014.40

[12] Gomes, A.C., Bueno, A.A., de Souza, R.G.M. and Mota, J.F. (2014) Gut Microbiota, Probiotics and Diabetes. Nutrition Journal, 13, Article No.: 60. https://doi.org/10.1186/1475-2891-13-60

[13] Louis, P., Hold, G.L. and Flint, H.J. (2014) The Gut Microbiota, Bacterial Metabolites and Colorectal Cancer. Nature Reviews Microbiology, 12, 661-672. https://doi.org/10.1038/nrmicro3344

[14] Scher, J.U., Ubeda, C., Artacho, A., et al. (2015) Decreased Bacterial Diversity Characterizes the Altered Gut Microbiota in Patients with Psoriatic Arthritis, Resembling Dysbiosis in Inflammatory Bowel Disease. Arthritis \& Rheumatology, 67, 128-139. https://doi.org/10.1002/art.38892

[15] Damman, C.J. and Surawicz, C.M. (2009) The Gut Microbiota: A Microbial Arsenal Protecting US from Infectious and Radiation-Induced Diarrhea. Gastroenterology, 136, 722-724. https://doi.org/10.1053/j.gastro.2008.12.020

[16] Collins, S.M., SureRe, M. and Bercik, E. (2012) The Interplay between the Intestinal Microbiota and the Brain. Nature Reviews Microbiology, 10, 735-742. https://doi.org/10.1038/nrmicro2876

[17] Cryan, J.F. and Dinan, T.G. (2012) Mind-Altering Microorganisms: The Impact of the Gut Microbiota on Brain and Behaviour. Nature Reviews Neuroscience, 13, 701-712. https://doi.org/10.1038/nrn3346

[18] Foster, J.A. and McVey Neufeld, K.-A. (2013) Gut Brain Axis: How the Microbiome Influences Anxiety and Depression. Trends in Neurosciences, 36, 305-312. https://doi.org/10.1016/j.tins.2013.01.005

[19] Verdu, E.F. (2009) Probiotics Effects on Gastrointestinal Function: Beyond the Gut? Neurogastroenterology and Motility, 21, 477-480. https://doi.org/10.1111/j.1365-2982.2009.01297.x

[20] Zhang, C., Yin, A., Zhao, L., et al. (2015) Dietary Modulation of Gut Microbiota Contributes to Alleviation of Both Genetic and Simple Obesity in Children. EBioMedicine, 2, 968-984. https://doi.org/10.1016/j.ebiom.2015.07.007

[21] Xu, J., Lian, F., Zhao, L., et al. (2015) Structural Modulation of Gut Microbiota during Alleviation of Type 2 Diabetes with a Chinese Herbal Formula. The ISME Journal, 9, 552-562. https://doi.org/10.1038/ismej.2014.177

[22] Qin, N., Yang, F.L., Li, A., et al. (2014) Alterations of the Human Gut Microbiome in Liver Cirrhosis. Nature, 513, 59-64. https://doi.org/10.1038/nature13568

[23] Sherwin, E. (2016) A Gut (Microbiome) Feeling about the Brain. Current Opinion in Gastroenterology, 32, 96-102. https://doi.org/10.1097/MOG.0000000000000244

[24] Naseribafrouei, A., Hestad, K., Avershina, E., et al. (2014) Correlation between the Human Fecal Microbiota and Depression. Neurogastroenterology \& Motility, 26, 1155-1162. https://doi.org/10.1111/nmo.12378

[25] Wang, Y. and Kasper, L.H. (2014) The Role of Microbiome in Central Nervous System Disorders. Brain, Behavior, and Immunity, 38, 1-12. https://doi.org/10.1016/j.bbi.2013.12.015

[26] Kelly, J.R., Borre, Y., O’Brien, C., et al. (2016) Transferring the Blues: Depression-Associated Gut Microbiota Induces Neurobehav-Ioural Changes in the Rat. Journal of Psychiatric Research, 82, 109-118.

[27] Park, A.J., Collins, J., Blennerhassett, P.A., et al. (2013) Altered Colonic Function and Microbiota Profile in a Mouse Model of Chronic Depression. Neurogastroenterology \& Motility, 25, 733-575. https://doi.org/10.1111/nmo.12153 
[28] Bailey, A.G., Dowd, S.E., Galley, J.D., et al. (2007) The Origin and Application of Experimental Autoimmune Encephalomyelitis. Nature Reviews Immunology, 7, 904-912. https://doi.org/10.1038/nri2190

[29] Svenaeus, F. (2013) Diagnosing Mental Disorders and Saving the Normal: American Psychiatric Association, 2013. Diagnostic and Statistical Manual of Mental Disorders. 5th Edition, American Psychiatric Publishing, Washington DC.

[30] Collins, S.M. (2014) A Role for the Gut Microbiota in IBS. Nature Reviews Gastroenterology \& Hepatology, 11, 497-505. https://doi.org/10.1038/nrgastro.2014.40

[31] He, Y., Wu, W., Zheng, H.M., et al. (2018) Regional Variation Limits Applications of Healthy Gut Microbiome Reference Ranges and Disease Models. Nature Medicine, 24, 1532-1535.

[32] Bambling, M., Edwards, S.C. and Hall, S. (2017) A Combination of Probiotics and Magnesium Orotate Attenuate Depression in a Small SSRI Resistant Cohort: An Intestinal Anti-Inflammatory Response Is Suggested. Inflammopharmacology, 25, 271-274. https://doi.org/10.1007/s10787-017-0311-x

[33] Parashar, A. and Udayabanu, M. (2016) Gut Microbiota Regulates Key Modulators of Social Behavior European Neuropsychopharmacology, 26, 78-91.

https://doi.org/10.1016/j.euroneuro.2015.11.002

[34] Bomyea, J., Risbrough, V. and Lang, A.J. (2012) A Consideration of Select Pre-Trauma Factors as Key Vulnerabilities in PTSD. Clinical Psychology Review, 32, 630-641. https://doi.org/10.1016/j.cpr.2012.06.008

[35] Bicanic, I.A., Postma, R.M., Sinnema, G., et al. (2013) Salivary Cortisol and Dehydroepiandrosterone Sulfate in Adolescent Rape Victims with Post Traumatic Stress Disorder. Psychoneuroendocrinology, 38, 408-415. https://doi.org/10.1016/j.psyneuen.2012.06.015

[36] Blier, P. (2013) Neurotransmitter Targeting in the Treatment of Depression. The Journal of Clinical Psychiatry, 74, 19-24. https://doi.org/10.4088/JCP.12084su1c.04

[37] Fetissov, S.O. and Déchelotte, P. (2011) The New Link between Gut-Brain Axis and Neuropsychiatric Disorders. Clinical Nutrition and Metabolic Care, 14, 477-482. https://doi.org/10.1097/MCO.0b013e32834936e7

[38] Fond, G., Loundou, A., Hamdani, N., et al. (2014) Anxiety and Depression Comorbidities in Irritable Bowel Syndrome (IBS): A Systematic Review and Meta-Analysis. European Archives of Psychiatry and Clinical Neuroscience, 264, 651-660. https://doi.org/10.1007/s00406-014-0502-Z

[39] Hungin, A.P., Becher, A., Cayley, B., et al. (2015) Irritable Bowel Syndrome: An Integrated Explanatory Model for Clinical Practice. Neurogastroenterology and Motility, 27, 750-763.

[40] Fetissov, S.O. and Déchelotte, P. (2011) The New Link between Gut-Brain Axis and Neuropsychiatric Disorders. Current Opinion in Clinical Nutrition and Metabolic Care, 14, 477-482. https://doi.org/10.1097/MCO.0b013e32834936e7

[41] Tak, L.M., Cleare, A.J., Ormel, J., et al. (2011) Meta-Analysis and Meta-Regression of Hypothalamic-Pituitary-Adrenal Axis Activity in Functional Somatic Disorders. Biological Psychology, 87, 183-194. https://doi.org/10.1016/j.biopsycho.2011.02.002

[42] Kim, J.Y., Kim, N., Seo, P.J., et al. (2013) Association of Sleep Dysfunction and Emotional Status with Gastroesophageal Reflux Disease in Korea. Journal of Neurogastroenterology and Motility, 19, 344-354. https://doi.org/10.5056/jnm.2013.19.3.344

[43] Hegeman, J.M., Kok, R.M., Van der Mast, R.C., et al. (2012) Phenomenology of De- 
pression in Older Compared with Younger Adults: Meta-Analysis. The British Journal of Psychiatry, 200, 275-281. https://doi.org/10.1192/bjp.bp.111.095950

[44] McElhanon, B.O., McCracken, C., Karpen, S., et al. (2014) Gastrointestinal Symptoms in Autism Spectrum Disorder: A Meta-Analysis. Pediatrics, 133, 872-883. https://doi.org/10.1542/peds.2013-3995

[45] Sandier, R.H., Finegold, S.M., Bolte, E.R., et al. (2000) Short-Term Benefit from Oral Vancomycin Treatment of Regressive-Onset Autism. Journal of Child Neurology, 15, 429-435. https://doi.org/10.1177/088307380001500701

[46] Bolte, E.R. (1998) Autism and Clostridium Tetani. Medical Hypotheses, 51, 133-144. https://doi.org/10.1016/S0306-9877(98)90107-4

[47] Finegold, S.M., Molitoris, D., Song, Y., et al. (2002) Gastrointestinal Microflora Studies in Late-Onset Autism. Clinical Infectious Diseases, 35, S6-S16. https://doi.org/10.1086/341914

[48] Finegold, S.M., Dowd, S.E., Gontcharova, V., et al. (2010) Pyrosequencing Study of Fecal Microflora of Autistic and Control Children. Anaerobe, 16, 444-453. https://doi.org/10.1016/j.anaerobe.2010.06.008

[49] Williams, B.L., Hornig, M., Buie, T., et al. (2011) Impaired Carbohydrate Digestion and Transport and Mucosal Dysbiosis in the Intestines of Children with Autism and Gastrointestinal Disturbances. PloS ONE, 6, e24585. https://doi.org/10.1371/journal.pone.0024585

[50] Wang, L., Christophersen, C.T., Sorich, M.J., Gerber, J.P., Angley, M.T. and Conlon, M.A. (2012) Elevated Fecal Short Chain Fatty Acid and Ammonia Concentrations in Children with Autism Spectrum Disorder. Digestive Diseases and Sciences, 57, 2096-2102. https://doi.org/10.1007/s10620-012-2167-7

[51] Adams, J.B., Johansen, L.J., Powell, L.D., Quig, D. and Rubin, R.A. (2011) Gastrointestinal Flora and Gastrointestinal Status in Children with Autism-Comparisons to Typical Children and Correlation with Autism Severity. BMC Gastroenterology, 11, Article No.: 22. https://doi.org/10.1186/1471-230X-11-22

[52] Berk, M., Williams, L.J., Jacka, F.N., et al. (2013) So Depression Is an Inflammatory Disease, but Where Does the Inflammation Come from? BMC Medicine, 11, Article No.: 200. https://doi.org/10.1186/1741-7015-11-200

[53] Gareau, M.G., Jury, J., MacQueen, G., Sherman, P.M. and Perdue, M.H. (2007) Probiotic Treatment of Rat Pups Normalises Corticosterone Release and Ameliorates Colonic Dysfunction Induced by Matemal Separation. Gut, 56, 1522-1528.

[54] Bravo, J.A., Forsythe, P., Chew, M.V., et al. (2011) Ingestion of Lactobacillus Strain Regulates Emotional Behavior and Central GABA Receptor Expression in a Mouse via the Vagus Nerve. Proceedings of the National Academy of Sciences of the United States of America, 108, 16050-16055. https://doi.org/10.1073/pnas.1102999108

[55] Desbonnet, L., Garrett, L., Clarke, G., Bienenstock, J. and Dinah, T.G. (2008) The Probiotic Bifidobacteria infantis. An Assessment of Potential Antidepressant Properties in the Rat. Journal of Psychiatric Research, 43, 164-174. https://doi.org/10.1016/j.jpsychires.2008.03.009

[56] Gundersen, B.B. and Blendy, J.A. (2009) Effects of the Histone Deacetylase Inhibitor Sodium Butyrate in Models of Depression and Anxiety. Neuropharmacology, 57, 67-74. https://doi.org/10.1016/j.neuropharm.2009.04.008

[57] Diaz Heijtz, R., Wang, S., Anuar, F., et al. (2011) Normal Gut Microbiota Modulates Brain Development and Behavior. Proceedings of the National Academy of Sciences of the United States of America, 108, 3047-3052.

https://doi.org/10.1073/pnas.1010529108 
[58] Clarke, G., Grenham, S., Scully, P., et al. (2013) The Microbiome-Gut-Brain Axis during Early Life Regulates the Hippocampal Serotonergic System in a Sex-Dependent Manner. Molecular Psychiatry, 18, 666-673. https://doi.org/10.1038/mp.2012.77

[59] Neufeld, K.M., Kang, N., Bienenstock, J. and Foster, J.A. (2011) Reduced Anxiety-Like Behavior and Central Neurochemical Change in Germ-Free Mice. Neurogastroenterology and Motility, 23, 255-e119. https://doi.org/10.1111/j.1365-2982.2010.01620.x

[60] Gaykema, R.P., Goehler, L.E. and Lyte, M. (2004) Brain Response to Cecal Infection with Campylobacter jejuni: Analysis with Fos Immunohistochemistry. Brain, Behavior, and Immunity, 18, 238-245. https://doi.org/10.1016/j.bbi.2003.08.002

[61] Bercik, P., Verdu, E.F., Foster, J.A., et al. (2010) Chronic Gastrointestinal Inflammation Induces Anxiety-Like Behavior and Alters Central Nervous System Biochemistry in Mice. Gastroenterology, 139, 2102-2112.e1.

https://doi.org/10.1053/j.gastro.2010.06.063

[62] Sudo, N., Chida, Y., Aiba, Y., et al. (2004) Postnatal Microbial Colonization Programs the Hypothalamic-Pituitary-Adrenal System for Stress Response in Mice. The Journal of Physiology, 558, 263-275. https://doi.org/10.1113/jphysiol.2004.063388

[63] O’Malley, D., Quigley, E.M., Dinan, T.G. and Cryan, J.E. (2011) Do Interactions between Stress and Immune Responses Lead to Symptom Exacerbations in Irritable Bowel Syndrome? Brain, Behavior, and Immunity, 25, 1333-1341. https://doi.org/10.1016/j.bbi.2011.04.009

[64] Zareie, M., Johnson-Henry, K., Jury, J., et al. (2006) Probiotics Prevent Bacterial Translocation and Improve Intestinal Barrier Function in Rats Following Chronic Psychological Stress. Gut, 55, 1553-1560.

[65] Ait-Belgnaoui, A., Durand, H., Cartier, C., et al. (2012) Prevention of Gut Leakiness by a Probiotic Treatment Leads to Attenuated HPA Response to an Acute Psychological Stress in Rats. Psychoneuroendocrinology, 37, 1885-1895. https://doi.org/10.1016/j.psyneuen.2012.03.024

[66] Bercik, P., Park, A.J., Sinclair, D., et al. (2011) The Anxiolytic Effect of Bifidobactefium longum NCC3001 Involves Vagal Pathways for Gut-Brain Communication. Neurogastroenterology and Motility, 23, 32-39. https://doi.org/10.1111/j.1365-2982.2011.01796.x 